\title{
ON THE INERTIA SETS OF SOME SYMMETRIC SIGN PATTERNS
}

\author{
C. M. DA Fonseca, Coimbra
}

(Received March 17, 2004)

Abstract. A matrix whose entries consist of elements from the set $\{+,-, 0\}$ is a sign pattern matrix. Using a linear algebra theoretical approach we generalize of some recent results due to Hall, $\mathrm{Li}$ and others involving the inertia of symmetric tridiagonal sign matrices.

Keywords: inertia, sign pattern matrix, tridiagonal matrix

MSC 2000: 15A18, 15A48

\section{INTRODUCTION}

Several authors have studied properties of a matrix based on combinatorial and qualitative information such as the signs of entries in the matrix. A matrix whose entries are from the set $\{+,-, 0\}$ is called a sign pattern matrix (or simply, sign pattern). For each $n \times n$ sign pattern $A$ there is a natural class of real matrices whose entries have the signs indicated by $A$, i.e., the sign pattern class of a sign pattern $A$ is defined by

$$
Q(A)=\{B ; \operatorname{sign} B=A\} .
$$

We are interested in symmetric matrices and in the sign symmetric classes

$$
Q_{\mathrm{SYM}}(A)=\left\{B ; \operatorname{sign} B=A \text { and } B=B^{T}\right\} .
$$

Define the inertia of an $n \times n$ real symmetric matrix $H$ as the triple $\operatorname{In}(H)=$ $(\pi, \nu, \delta)$, where $\pi$ is the number of positive eigenvalues, $\nu$ is the number of negative eigenvalues and $\delta=n-\pi-\nu$ is the number of zero eigenvalues. For a symmetric sign pattern $A$, we define the inertia (set) of $A$ to be $\operatorname{In}(A)=\left\{\operatorname{In}(B) ; B \in Q_{\mathrm{SYM}}(A)\right\}$.

This work was supported by CMUC - Centro de Matemática da Universidade de Coimbra. 
We say the sign pattern $A$ requires unique inertia and is sign nonsingular if every real matrix in $Q(A)$ has the same inertia and is nonsingular, respectively. If two sign patterns $A_{1}$ and $A_{2}$ are congruent, i.e., if for all $B_{1} \in Q_{\mathrm{SYM}}\left(A_{1}\right)$ and $B_{2} \in Q_{\mathrm{SYM}}\left(A_{2}\right)$ there exists a nonsingular real matrix $S$ such that $B_{1}=S B_{2} S^{T}$, then we say that $A_{1}$ and $A_{2}$ are sign congruent and write $A_{1} \approx A_{2}$.

By Sylvester's law of inertia we may say that two sign congruent patterns have the same inertia. For example, the symmetric sign pattern

$$
\left[\begin{array}{ccc}
0 & + & + \\
+ & 0 & + \\
+ & + & 0
\end{array}\right]
$$

is sign congruent to

$$
\left[\begin{array}{ccc}
0 & + & 0 \\
+ & 0 & 0 \\
0 & 0 & -
\end{array}\right]
$$

and, therefore, requires the unique inertia $(1,2,0)$ and, consequently, is sign nonsingular. On the other hand, the tridiagonal sign pattern

$$
\left[\begin{array}{lll}
+ & + & 0 \\
+ & + & + \\
0 & + & +
\end{array}\right]
$$

is sign congruent to

$$
\left[\begin{array}{ccc}
+ & 0 & 0 \\
0 & * & 0 \\
0 & 0 & +
\end{array}\right],
$$

where $*$ is $0,+$ or - , and, therefore, requires the inertias $(2,0,1),(3,0,0)$, and $(2,1,0)$.

A diagonal sign pattern each of whose entries is + or - is called a signature pattern. The square of a signature pattern is a signature pattern with all nonzero entries equal to + . A sign pattern such that there is exactly one entry in each row and each column equal to + and all other entries are 0 is called a permutation pattern. Two sign congruent patterns by the way of a signature pattern and of a permutation pattern are called, respectively, signature congruent and permutation congruent patterns.

In this paper we generalize recent results on some symmetric sign patterns due to F. Hall, Z. Li and others (cf. [3], [4], [6], [7]). The results of these authors are based on a graph theoretical approach. Here we use mainly tools from congruences between matrices developed, e.g., by B. Cain and E. Marques de Sá (cf. [1], [2]). 


\section{Symmetric tridiagonal SIgN PATtERnS}

Given a symmetric tridiagonal sign pattern, the inertia does not depend on the sign of the off-diagonal elements, since two sign patterns under these conditions are signature congruent. Let us denote these entries by \pm .

The symmetric tridiagonal sign pattern

(1)

$$
\left[\begin{array}{ccccccc}
0 & \pm & & & & & \\
\pm & * & \pm & & & & \\
& \pm & 0 & \pm & & & \\
& & \pm & * & \pm & & \\
& & & \pm & 0 & \pm & \\
& & & & \ddots & \ddots & \ddots
\end{array}\right]_{n \times n}
$$

is congruent to

$$
\left[\begin{array}{ccccccc}
0 & \pm & & & & & \\
\pm & * & 0 & & & & \\
& 0 & 0 & \pm & & & \\
& & \pm & * & 0 & & \\
& & & 0 & 0 & \pm & \\
& & & & \ddots & \ddots & \ddots
\end{array}\right]
$$

i.e., it is congruent to the direct sum

$$
\left[\begin{array}{ll}
0 & \pm \\
\pm & *
\end{array}\right] \oplus \ldots \oplus\left[\begin{array}{ll}
0 & \pm \\
\pm & *
\end{array}\right] \oplus[0]
$$

if $n$ is odd, and to

$$
\left[\begin{array}{ll}
0 & \pm \\
\pm & *
\end{array}\right] \oplus \ldots \oplus\left[\begin{array}{ll}
0 & \pm \\
\pm & *
\end{array}\right]
$$

if $n$ is even. Since the inertia of each block

$$
\left[\begin{array}{ll}
0 & \pm \\
\pm & *
\end{array}\right]
$$

is $(1,1,0)$, we can generalize now Proposition 3.1 in $[6]$. 
Proposition 2.1. For the symmetric tridiagonal sign pattern defined in (1),

(a) if $n$ is even, then $A$ is sign nonsingular and $\operatorname{In}(A)=\left(\frac{n}{2}, \frac{n}{2}, 0\right)$,

(b) if $n$ is odd, then $A$ is sign singular and $\operatorname{In}(A)=\left(\frac{n-1}{2}, \frac{n-1}{2}, 1\right)$.

Note that the above proposition is still true for the $n \times n$ sign pattern

$$
\left[\begin{array}{ccccccc}
* & \pm & & & & & \\
\pm & 0 & \pm & & & & \\
& \pm & * & \pm & & & \\
& & \pm & 0 & \pm & & \\
& & & \pm & * & \pm & \\
& & & & \ddots & \ddots & \ddots
\end{array}\right]
$$

provided $n$ is even.

Let us consider the $n \times n$ sign pattern

$$
\left[\begin{array}{cccc}
+ & \pm & & \\
\pm & + & \pm & \\
& \pm & + & \ddots \\
& & \ddots & \ddots
\end{array}\right]
$$

With the + in the $(1,1)$-entry we can, by congruence operations, "eliminate" the off-diagonals entries $(1,2)$ and $(2,1)$. If the new $(2,2)$-entry is 0 and $n>2$, then we can decompose the sign pattern so that the first block is

$$
\left[\begin{array}{ccc}
+ & 0 & \\
0 & 0 & \pm \\
& \pm & 0
\end{array}\right],
$$

which has inertia $(2,1,0)$. In the case of $n=2$, the block is simply

$$
\left[\begin{array}{cc}
+ & 0 \\
0 & 0
\end{array}\right]
$$

which has inertia $(1,0,1)$. If the new $(2,2)$-entry is a - , then we can decompose the sign pattern so that the first block is

$$
\left[\begin{array}{cc}
+ & 0 \\
0 & -
\end{array}\right]
$$

which has inertia $(1,1,0)$. The new $(3,3)$-entry is always a + and we restart the procedure from here.

Otherwise, the $(2,2)$-entry is a + , and the first block of the composition is simply $[+]$ and we restart the procedure from that entry.

By the above algorithm we can establish the maxima and minima for the number of eigenvalues. 


\section{Proposition 2.2. If}

$$
A_{+}=\left[\begin{array}{ccccc}
+ & \pm & & & \\
\pm & + & \pm & & \\
& \pm & \ddots & \ddots & \\
& & \ddots & \ddots & \pm \\
& & & \pm & +
\end{array}\right]
$$

is an $n \times n$ symmetric tridiagonal sign pattern, then $\operatorname{In}\left(A_{ \pm}\right)$has the form

$$
(n-k, k, 0), 0 \leqslant k \leqslant\left\lfloor\frac{n}{2}\right\rfloor, \quad \text { or } \quad(n-k, k-1,1), 1 \leqslant k \leqslant\left\lfloor\frac{n}{2}\right\rfloor,
$$

where $\lfloor x\rfloor$ denotes the greater integer less than or equal to the real number $x$.

Given a sign pattern we say that the diagonal $(i, i)$-entry is in an odd (even) position when $i$ is odd (even). The diagonal $(i, i)$ and $(j, j)$-entries are said to be in ascending positions provided $i<j$ (not necessarily consecutive).

We can rewrite some results from [6] and [7], generalize them and give a straightforward proof.

Theorem 2.3. For the symmetric tridiagonal sign pattern

$$
A_{*}=\left[\begin{array}{ccccc}
* & \pm & & & \\
\pm & * & \pm & & \\
& \pm & \ddots & \ddots & \\
& & \ddots & \ddots & \pm \\
& & & \pm & *
\end{array}\right]
$$

where each diagonal entry is $0,+$ or - ,

(a) if $n$ is even, then $A_{*}$ is sign nonsingular if and only if neither two + nor two diagonal entries in $A_{*}$ are in odd-even ascending positions, respectively. In this case $\operatorname{In}\left(A_{*}\right)=\left(\frac{n}{2}, \frac{n}{2}, 0\right)$;

(b) if $n$ is odd, then $A_{*}$ is sign nonsingular if there is at least one + or - diagonal entry in an odd position, but not both in odd positions, and neither three + nor three - diagonal entries are in odd-even-odd ascending positions, respectively. In this case $\operatorname{In}\left(A_{*}\right)=\left(\frac{n+1}{2}, \frac{n-1}{2}, 0\right)$ if there are + in odd positions, or $\operatorname{In}\left(A_{*}\right)=$ $\left(\frac{n-1}{2}, \frac{n+1}{2}, 0\right)$ if there are - in odd positions.

Proof. Suppose that $n$ is even. Without loss of generality we may assume that the first and the last diagonal entries are non-zero. In order for $A_{*}$ to require 
unique inertia, when we use congruence relations in order to eliminate the off-diagonal elements, the signs of the diagonal should alternate between + and - .

By Proposition 2.1, if $n$ is odd and neither + nor - diagonal entries are in odd positions, then $A_{*}$ requires unique inertia $\left(\frac{n-1}{2}, \frac{n-1}{2}, 1\right)$. Without loss of generality we may assume that the first diagonal entry is non-zero. Assume that it is a + . Then using the congruence elimination procedure, we can not have - in odd diagonal positions and no three + diagonal entries in odd-even-odd ascending positions.

The sign pattern

$$
\left[\begin{array}{cccccc}
+ & + & & & & \\
+ & - & - & & & \\
& - & 0 & + & & \\
& & + & 0 & + & \\
& & & + & + & - \\
& & & & - & 0
\end{array}\right]
$$

is congruent to

$$
[+] \oplus[-] \oplus\left[\begin{array}{cc}
0 & + \\
+ & 0
\end{array}\right] \oplus\left[\begin{array}{cc}
0 & - \\
- & 0
\end{array}\right]
$$

and, therefore, requires unique inertia $(3,3,0)$.

However, the sign pattern

$$
\left[\begin{array}{ccccccc}
+ & + & & & & \\
+ & - & - & & & \\
& - & 0 & + & & \\
& & + & + & + & \\
& & & + & + & - \\
& & & & - & 0
\end{array}\right]
$$

is congruent to

$$
[+] \oplus[-] \oplus[+] \oplus[*] \oplus\left[\begin{array}{cc}
0 & - \\
- & 0
\end{array}\right]
$$

and the inertia set is $\{(3,2,1),(4,2,0),(3,3,0)\}$.

Let us give another example. The sign pattern

$$
\left[\begin{array}{cccccccc}
+ & + & & & & & \\
+ & - & - & & & & \\
& - & + & + & & & \\
& & + & 0 & + & & \\
& & & + & 0 & - & \\
& & & & - & - & + \\
& & & & & & + & +
\end{array}\right]
$$


is sign congruent to

$$
\left[\begin{array}{lllllll}
+ & & & & & & \\
& - & & & & \\
& & + & & & \\
& & - & & \\
& & & + & & \\
& & & & - & \\
& & & & & +
\end{array}\right]
$$

and hence requires unique inertia $(4,3,0)$, but the sign pattern

$$
\left[\begin{array}{ccccccc}
+ & + & & & & & \\
+ & - & - & & & & \\
& - & + & + & & & \\
& & + & 0 & + & & \\
& & & + & - & - & \\
& & & & - & - & + \\
& & & & & + & +
\end{array}\right]
$$

is congruent to

$$
[+] \oplus[-] \oplus[+] \oplus[-] \oplus[*] \oplus[-] \oplus[+]
$$

and the inertia set is $\{(3,3,1),(4,3,0),(3,4,0)\}$.

\section{Symmetric StAR SIGN PATterns}

We now consider a symmetric tree sign pattern matrix whose associated graph is a star.

Theorem 3.1 [7]. Up to permutation congruence, signature congruence, and negation, a symmetric star sign pattern

$$
A=\left[\begin{array}{ccccc}
* & + & + & \ldots & + \\
+ & * & & & \\
+ & & * & & \\
\vdots & & & \ddots & \\
+ & & & & *
\end{array}\right]_{n \times n}
$$

where each diagonal entry is $0,+$ or - , requires unique inertia if and only if the diagonal of $A$ has the following forms:

$$
(*, \ldots, *, 0),(0,+, \ldots,+),(-,+, \ldots,+) .
$$


Pro of. With the exception of the $(1,1)$-entry, if one of the diagonal entries is zero, then

$$
A \approx\left[\begin{array}{cc}
0 & + \\
+ & 0
\end{array}\right] \oplus\left[\begin{array}{lll}
* & & \\
& \ddots & \\
& & *
\end{array}\right]_{n-2 \times n-2},
$$

and $A$ requires unique inertia.

Suppose now that all the diagonal entries are nonzero, possibly with the exception of the (1,1)-entry. Then

$$
A \approx[*] \oplus\left[\begin{array}{lll}
* & & \\
& \ddots & \\
& & *
\end{array}\right]_{n-1 \times n-1} .
$$

In this case, $A$ requires unique inertia if and only if all the diagonal entries different from the $(1,1)$-entry have the same sign and the $(1,1)$-entry has a sign different from the other diagonal elements or is equal to 0 .

\section{Sign patterns With ALL + OFF-Diagonal Entries}

Finally, let $J_{n}$ be the $n \times n$ symmetric sign pattern with all entries equal to + . Then

$$
J_{n} \approx[+] \oplus B,
$$

where $B$ is a symmetric sign pattern of order $n-1$. Then the set of possible inertias of $J_{n}$ is

$$
\{(\pi, \nu, n-\pi-\nu) ; 1 \leqslant \pi \leqslant n, \pi+\nu \leqslant n\} .
$$

If one considers $\hat{J}_{n}$, the $n \times n$ symmetric sign pattern with zero diagonal and + off-diagonal entries, then

$$
\hat{J}_{n} \approx\left[\begin{array}{ccc}
0 & + & 0 \\
+ & 0 & 0 \\
0 & 0 & -
\end{array}\right] \oplus B,
$$

where $B$ is a symmetric sign pattern of order $n-3$. Therefore the set of possible inertia of $\hat{J}_{n}$ is

$$
\{(\pi, \nu, n-\pi-\nu) ; 1 \leqslant \pi \leqslant n, 2 \leqslant \nu \leqslant n, \pi+\nu \leqslant n\} .
$$

This last result was obtained recently by Gao and Shao [5] via a different approach.

Acknowledgment. This work was supported by CMUC - Centro de Matemática da Universidade de Coimbra. 


\section{References}

[1] B. E. Cain and E. Marques de Sá: The inertia of Hermitian matrices with a prescribed $2 \times 2$ block decomposition. Linear and Multilinear Algebra 31 (1992), 119-130.

Zbl 0756.15024

[2] B. E. Cain and E. Marques de Sá: The inertia of certain skew-triangular block matrices. Linear Algebra Appl. 160 (1992), 75-85.

Zbl 0752.15006

[3] C. Eschenbach and C.R. Johnson: A combinatorial converse to the Perron-Frobenius theorem. Linear Algebra Appl. 136 (1990), 173-180. Zbl 0739.15004

[4] C. Eschenbach and C.R. Johnson: Sign patterns that require real, nonreal or pure imaginary eigenvalues. Linear and Multilinear Algebra 29 (1991), 299-311. Zbl 0764.05015

[5] Y. Gao and Y. Shao: The inertia set of nonnegative symmetric sign pattern with zero diagonal. Czechoslovak Math. J. 53 (2003), 925-934.

[6] F. J. Hall and Z. Li: Inertia sets of symmetric sign pattern matrices. Numer. Math. J. Chinese Univ. (English Ser.) 10 (2001), 226-240.

Zbl 0997.15010

[7] F. J. Hall, Z. Li and Di Wang: Symmetric sign pattern matrices that require unique inertia. Linear Algebra Appl. 338 (2001), 153-169. Zbl 0994.15028

[8] R.A.Horn and C.R. Johnson: Matrix Analysis, Cambridge University Press, Cambridge. 1985

Zbl 0576.15001

[9] C. Jeffries and C.R. Johnson: Some sign patterns that preclude matrix stability. SIAM J. Matrix Anal. Appl. 9 (1988), 19-25.

Zbl 0676.15004

Author's address: Departamento de Matemática, Universidade de Coimbra, 3001-454

Coimbra, Portugal, e-mail: cmf@mat.uc.pt. 